OPEN

SUBJECT AREAS:

NANOSCALE DEVICES

NANOPHOTONICS AND

PLASMONICS

Received

2 June 2014

Accepted

20 August 2014

Published

22 September 2014

Correspondence and requests for materials should be addressed to

R.R. (ranjith. rajasekharan@ unimelb.edu.au)

\title{
Filling schemes at submicron scale: Development of submicron sized plasmonic colour filters
}

\author{
Ranjith Rajasekharan', Eugeniu Balaur², Alexander Minovich ${ }^{3}$, Sean Collins ${ }^{4}$, Timothy D. James ${ }^{1,2}$, \\ Amir Djalalian-Assl' , Kumaravelu Ganesan', Snjezana Tomljenovic-Hanic' ', Sasikaran Kandasamy², \\ Efstratios Skafidas ${ }^{5}$, Dragomir N. Neshev ${ }^{3}$, Paul Mulvaney ${ }^{4}$, Ann Roberts' ${ }^{1}$ \& Steven Prawer ${ }^{1}$
}

\begin{abstract}
${ }^{1}$ School of Physics, The University of Melbourne, VIC 3010, Australia, ${ }^{2}$ Melbourne Centre for Nanofabrication, Australian National Fabrication Facility, Clayton, VIC, 3168, Australia, ${ }^{3}$ Nonlinear Physics Centre, Research School of Physics and Engineering, Australian National University, Canberra ACT 0200, Australia, ${ }^{4}$ School of Chemistry and Bio2 1 Institute, University of Melbourne Parkville, VIC 3010, Australia, ${ }^{5}$ Department of Electrical and Electronic Engineering, The University of Melbourne, Melbourne, VIC. 3010 , Australia.
\end{abstract}

The pixel size imposes a fundamental limit on the amount of information that can be displayed or recorded on a sensor. Thus, there is strong motivation to reduce the pixel size down to the nanometre scale.

Nanometre colour pixels cannot be fabricated by simply downscaling current pixels due to colour cross talk and diffraction caused by dyes or pigments used as colour filters. Colour filters based on plasmonic effects can overcome these difficulties. Although different plasmonic colour filters have been demonstrated at the micron scale, there have been no attempts so far to reduce the filter size to the submicron scale. Here, we present for the first time a submicron plasmonic colour filter design together with a new challenge - pixel boundary errors at the submicron scale. We present simple but powerful filling schemes to produce submicron colour filters, which are free from pixel boundary errors and colour cross- talk, are polarization independent and angle insensitive, and based on LCD compatible aluminium technology. These results lay the basis for the development of submicron pixels in displays, RGB-spatial light modulators, liquid crystal over silicon, Google glasses and pico-projectors.

\footnotetext{
C onventional colour filters are made of dyes or pigments and exploit their particular absorption properties to produce different colours in displays ${ }^{1-3}$. However, as the pixel size is reduced to a few microns, conventional colour filters start to suffer from colour cross talk ${ }^{4}$. Furthermore, RGB filters utilizing existing technology must be fabricated in several steps, which presents severe challenges when trying to accomplish submicron scale alignment ${ }^{3}$. Coupling light into submicron scale pixels is also extremely difficult due to diffraction. These issues call for new strategies for the fabrication of submicron sized colour pixels.

Colour filters based on plasmonic effects ${ }^{5-11}$ are superior to conventional filters, especially when the pixel size is reduced to a few microns in size. This is because dye based filters cannot be made much thinner than several hundred nanometres because of their low absorption coefficients and because fabrication of each of the three dye filters for RGB colour schemes demands complex lithographic processes ${ }^{5}$. A further advantage of plasmonic filters is that they are tunable across the entire visible spectrum using just a single, optically thick metal film. Plasmonic filters based on aluminium (Al) are highly desirable compared to gold and silver for submicron scale colour pixels because $\mathrm{Al}$ is inexpensive, compatible with existing LCD technology and has good adhesion to many substrates, obviating the need for deposition of an extra adhesion layer, thus simplifying fabrication ${ }^{12,13}$. There are other advantages to using $\mathrm{Al}$ such as its lower optical loss in the 400 to $500 \mathrm{~nm}$ spectral range, due to its high bulk plasma frequency ${ }^{13-15}$. In principle, plasmonic filters are recyclable, have reduced cross talk, are durable at high temperature and are resilient to prolonged exposure to ultraviolet radiation ${ }^{5,14,15}$. It is also possible to directly couple light into the structures when submicron scale plasmonic filters are used as colour pixels. Recently, micron-sized, hole-based, plasmonic filters of $\mathrm{Al}$ were shown to exhibit transmission coefficients of more than $35 \%{ }^{5}$. A hole-array based plasmonic filter design is superior to that based on slits or lines as the former is polarization independent ${ }^{15-20,22}$.
} 


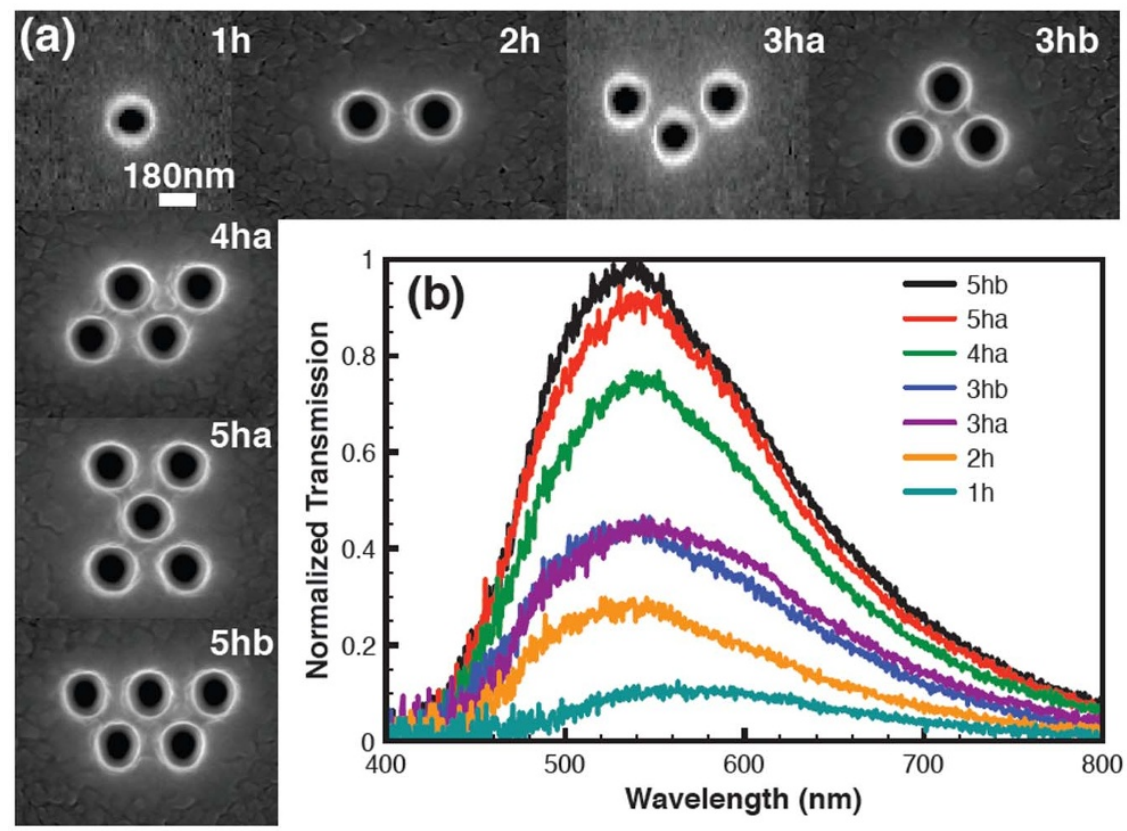

Figure 1 Investigation of the minimum number of holes required to define a submicron pixel (a) SEM images of a series of pixels composed of 1 to 5 holes in a hexagonal array (1h-5hb). Holes are $180 \mathrm{~nm}$ diameter with a $330 \mathrm{~nm}$ pitch fabricated in Al. (b) Experimental transmission spectra as a function of wavelength, showing the filtering effect as a function of the number of holes. An unpolarized white light source was used for the measurements.

However, the development of submicron scale plasmonic RGB colour filters in $\mathrm{Al}$ for display technology remains a major challenge because there are several stringent requirements that the submicron scale colour filters need to satisfy. They should be made of a single component film (for easy integration onto a display panel); they must be transparent in the visible region of the spectrum (must operate in transmission mode); they must be polarization independent (in order to maximize the transmission efficiency); they should also be angle insensitive (the filtered colour should be same for any angle of excitation); they should exhibit reduced colour crosstalk by avoiding multiple spectral transmission peaks and finally, each RGB pixel should have the same size at the submicron scale. Even if all the above criteria are met, the design of the submicron pixels for display applications is not possible unless pixel boundary errors are eliminated at the submicron scale.

Our study consists of five key steps: (i) finding the minimum plasmonic filter size required to define a submicron scale pixel using simple holes in aluminium; (ii) illustrating pixel boundary errors (or filter boundary errors) at the submicron scale; (iii) developing a filling scheme to eliminate the pixel boundary errors (iv) demonstrating a $430 \mathrm{~nm} \times 392 \mathrm{~nm}$ plasmonic filter (v) developing an angle insensitive submicron scale filter of size $690 \mathrm{~nm} \times 632 \mathrm{~nm}$ based on a coaxial hole-coaxial hole ( $\mathrm{CH}-\mathrm{CH})$ filling combination by tuning surface plasmons and Fabry-Perot resonances to eliminate pixel boundary errors.

\section{Results}

Investigation of minimum number of holes required to define a submicron scale colour filter. We start by finding the minimum plasmonic filter size required to define a submicron scale pixel using simple holes in aluminium. A hexagonal arrangement is used for the submicron filter design rather than a square arrangement because the wavelength interval between the first two surface plasmon resonance peaks in a hexagonal array is larger than that in a square array ${ }^{19-21}$. For a hexagonal arrangement, the distance from the central hole to the next row of holes is the same. Since the surface plasmon resonance is sensitive to the pitch, contributions from all holes add constructively. For a square array, the pitch is different in the diagonal direction. The difference in pitch causes multiple peaks and a reduction in the overall transmission intensity (see Supplementary Information Section.1 and Fig. S1 for details). We have fabricated from one to five holes with different geometries in $\mathrm{Al}$ films, which are deposited on a glass wafer (fabrication details are given in Methods). The hole diameter is chosen to be $180 \mathrm{~nm}$ and the array pitch $330 \mathrm{~nm}$ in order to create a resonance peak close to $540 \mathrm{~nm}$. Fig. 1b shows the variation in transmission peak intensity as a function of the number of holes and their geometry. These spectra have been collected from each of the single pixels shown in Figure 1. It is clear from the spectrum that colour filtering by a singlehole based pixel is broad and weak. Spectral filtering is already observed with two holes, as is evident from the slight increase in the transmission peak and the blue-shift induced by plasmon coupling. This is, however, not sufficient to produce a satisfactory pixel because there is strong polarization sensitivity due to the elongated shape of the pixel. For three holes, there are two arrangements possible. The first one is an L shaped arrangement of holes (3ha in Fig. 1a) while the second one is a triangular hole arrangement ( $3 h b$ in Fig. 1a). The transmission increases for both arrangements. It is interesting to note that the full width at half maximum (FWHM) for $3 h a$ is slightly larger than for $3 h b$ (Fig. 1a). For $3 h a$, an isosceles triangle arrangement is used while in $3 h b$, an equilateral triangle arrangement is utilized. Three-hole pixels clearly exhibit improved filtering, with transmission increasing from 0.1 (a.u) (for a single hole) to 0.45 (triple hole) and this pattern also gives a peak position at the designed wavelength $(530 \mathrm{~nm})$ as shown in Fig. 1. There is another obvious advantage to the equilateral triangle arrangement $(3 \mathrm{hb})$ : it is polarization independent due to its symmetry ${ }^{16}$.

For four holes, we have considered only one geometry (4ha in Fig. 1a), as other possible geometries are not useful for the pixel development due to their irregular morphologies and lower symmetry. For five holes, there are two likely candidates: a rectangular geometry (5ha in Fig. 1a) and an elongated geometry (5hb in Fig. 1a). The peak lies at $530 \mathrm{~nm}$ for both structures (the same as for the four hole pixel, but with slightly increased transmission). The elongated 

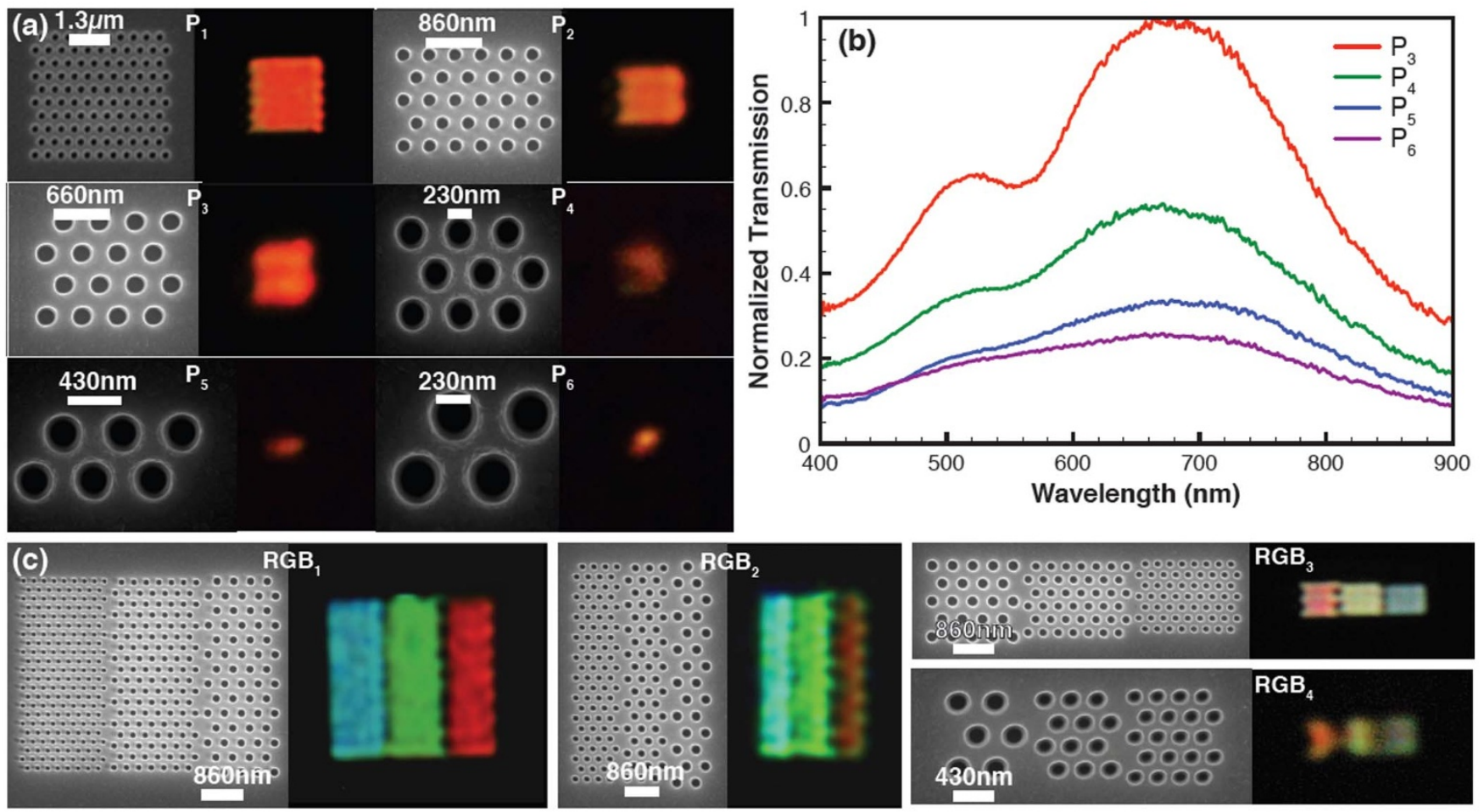

Figure $2 \mid$ Development of submicron colour filters (a) SEM images of red colour filters of different sizes and the corresponding optical images $\left(\mathrm{P}_{1}-\mathrm{P}_{6}\right)$ $(100 \times$ magnification). The filter size varies from $4.53 \mu \mathrm{m}$ down to $660 \mathrm{~nm}$. The optical images of the submicron scale plasmonic filters mimic the SEM images (pixel boundary errors) $\left(\mathrm{P}_{1}-\mathrm{P}_{6}\right)(\mathrm{b})$ Experimental transmission spectra of the red submicron filters, $\mathrm{P}_{3}(1.73 \mu \mathrm{m} \times 1.53 \mu \mathrm{m}), \mathrm{P}_{4}(1.3 \mu \mathrm{m} \times$ $975 \mathrm{~nm}), \mathrm{P}_{5}(1.3 \mu \mathrm{m} \times 602 \mathrm{~nm})$, and $\mathrm{P}_{6}(660 \mathrm{~nm} \times 602 \mathrm{~nm})$ display a peak at $680 \mathrm{~nm}(\mathrm{c})$ SEM images of submicron colour filters with sub-RGB filters. The sub-RGB filter sizes for the red are $1.95 \mu \mathrm{m} \times 5 \mu \mathrm{m}\left(\mathrm{RGB}_{1}\right), 875 \mathrm{~nm} \times 5 \mu \mathrm{m}\left(\mathrm{RGB}_{2}\right), 1.95 \mu \mathrm{m} \times 1.72 \mu \mathrm{m}\left(\mathrm{RGB}_{3}\right)$ and $875 \mathrm{~nm} \times 975 \mathrm{~nm}\left(\mathrm{RGB}_{4}\right)$. The corresponding optical images $\left(100 \times\right.$ magnification) display pixel stitching errors at the boundaries $\left(\mathrm{RGB}_{1}-\mathrm{RGB}_{4}\right)$.

$5 h b$ pixel has a higher transmission than the $5 h a$, which again highlights the importance of optimizing the hole format for the pixels. We conclude that, after taking into account the transmission, line-width, minimum size and polarization insensitiveness, the minimum number of holes required for realizing a submicron colour pixel is three in a triangular arrangement as shown in Fig. 1 (a), $3 \mathrm{hb}$.

Next, we have investigated whether there is any major shift in the peak wavelength position when the plasmonic filter size is reduced to submicron size. A red plasmonic filter with different sizes is fabricated (the size is varied from $4.53 \mu \mathrm{m}$ to $660 \mathrm{~nm}$ ) for the study (optimization of parameters is given in the Supplementary Information Section. 2 and Fig. S2). Figure 2a shows SEM images of these red filters, along with the corresponding images obtained under an optical microscope in transmission mode with $100 \times$ magnification. The images and the related spectra confirm the successful operation of the red colour filter at a submicron scale. Fig. $2 \mathrm{~b}$ displays the transmission spectra obtained for filters $\mathrm{P}_{3}(1.73 \mu \mathrm{m} \times 1.53 \mu \mathrm{m})$, $\mathrm{P}_{4}(1.3 \mu \mathrm{m} \times 975 \mathrm{~nm}), \mathrm{P}_{5}(1.3 \mu \mathrm{m} \times 602 \mathrm{~nm})$, and $\mathrm{P}_{6}(660 \mathrm{~nm} \times$ $602 \mathrm{~nm})$. Surprisingly, the peak position of the plasmons resonance remains around $680 \mathrm{~nm}$ for all the filter sizes. Here, the transmission at shorter wavelengths is dominated by photonic modes supported by the holes. The photonic modes are due to strongly localized field distributions inside the hole itself ${ }^{5}$ and is the reason for the occurrence of further minor peaks at shorter wavelength evident in Fig. $2 b$. A coaxial hole geometry instead of a hole geometry could suppress these photonic modes ${ }^{5}$. This could be realised by a structure composed of concentric, cylindrical holes. The resonance in such a geometry is dominated by Fabry-Perot resonances.

Pixel boundary errors at the submicron scale. As demonstrated above, an equilateral triangular arrangement of holes defines the simplest, polarization-insensitive filter. Thus each plasmonic pixel should be triangular in geometry and is suitable for displays where triangular pixels are used. However, most of the current, state-of-theart display technologies utilise rectangular or square pixels. Realization of a rectangular or square filter is challenging when a triangular (hexagonal) arrangement of holes is used. From Fig. 2, the optical image of the submicron scale plasmonic filters follows its own SEM image and this is termed a pixel boundary error. It is clear that there are irregularities at the edges of the submicron filters, due to the hexagonal arrangement of holes - this makes the design of a rectangular or square submicron scale filter an enormous challenge. The errors are visible even for a filter size of $4.53 \mu \mathrm{m}$ as shown in Fig. $2 \mathrm{a}\left(\mathrm{P}_{1}\right)$. As the filter size decreases from $4.53 \mu \mathrm{m} \times$ $3.58 \mu \mathrm{m}$ to $660 \mathrm{~nm} \times 602 \mathrm{~nm}\left(\mathrm{P}_{1}-\mathrm{P}_{6}\right.$ in Fig. $\left.2 \mathrm{a}\right)$ the filter boundary errors become more prominent. For example, the optical image of the red filter evinces a crescent shape when the size is reduced to $1.3 \mu \mathrm{m} \times 975 \mathrm{~nm}$ as shown in the Fig. $2 \mathrm{a}\left(\mathrm{P}_{4}\right)$.

We have further studied the combined effect of RGB filters when submicron scale RGB filters are placed together. The submicron scale filters with sub-RGB filters of different colours are fabricated (see Methods) based on computational studies using a finite number of holes (Supplementary Information Section. 2 and Fig. S2). Figure 2c shows SEM images of the fabricated filters. When the same number of holes is used for making each sub-RGB filter, the filter size is different for each red, green and blue filter. This is due to the different pitches required to generate the three different colours. In order to keep each sub-RGB pixel size almost the same, we have adjusted the number of holes comprising the red, green and blue filters, as shown in Fig. 2c. The sub-RGB filter sizes for the red are $1.95 \mu \mathrm{m} \times 5 \mu \mathrm{m}$ $\left(\mathrm{RGB}_{1}\right), 875 \mathrm{~nm} \times 5 \mu \mathrm{m}\left(\mathrm{RGB}_{2}\right), 1.95 \mu \mathrm{m} \times 1.72 \mu \mathrm{m}\left(\mathrm{RGB}_{3}\right)$ and $875 \mathrm{~nm} \times 975 \mathrm{~nm}\left(\mathrm{RGB}_{4}\right)$. Fig. $2 \mathrm{c}$ also shows the corresponding optical images $(100 \times$ Magnification $)$. We have placed each filter with a separation of $40 \mathrm{~nm}$. The optical images reveal irregularities 

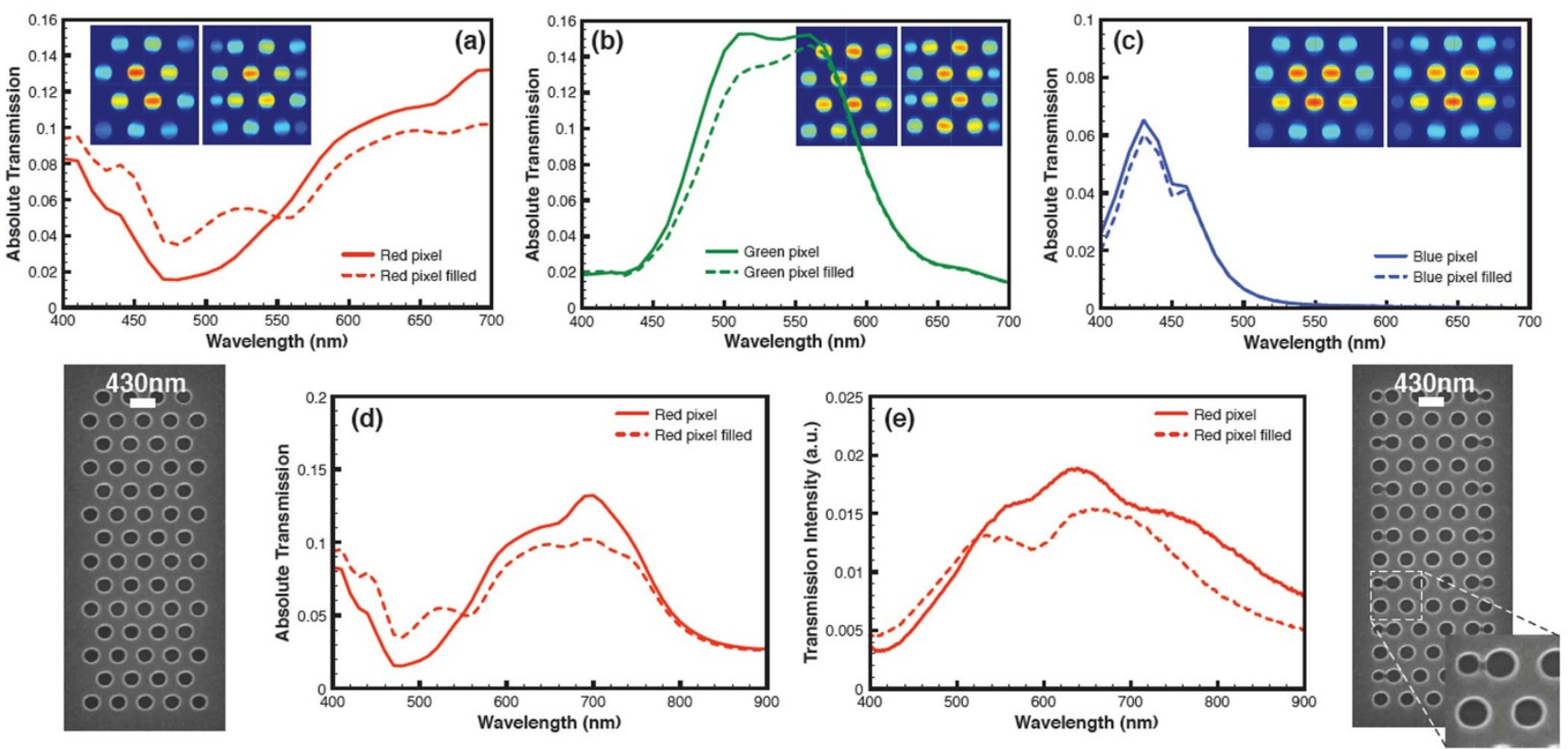

(f)
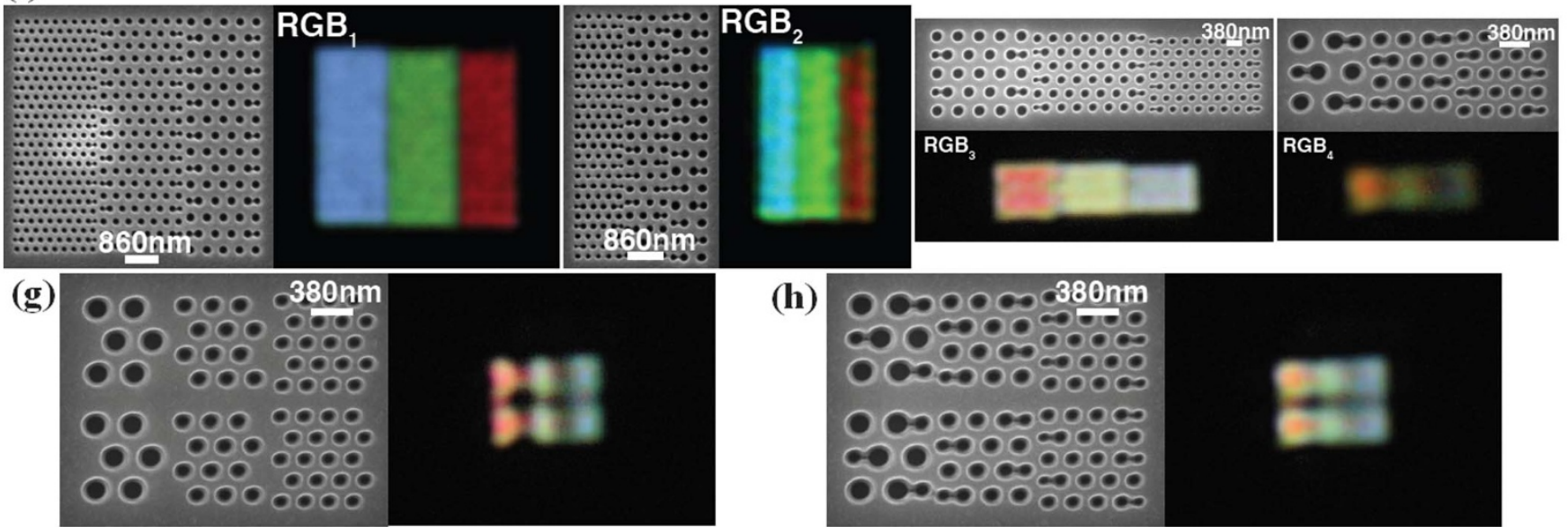

(h)

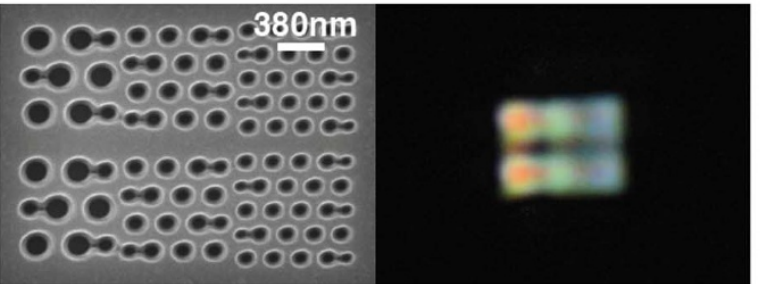

Figure 3 The pixel filling scheme. Numerically simulated transmission spectra before and after filling using $3 \times 4$ hole matrices for (a) a red filter (b) a green filter (c) a blue filter with wavelength swept from $400 \mathrm{~nm}$ to $700 \mathrm{~nm}$ (d) a red filter with wavelength swept from $400 \mathrm{~nm}$ to $900 \mathrm{~nm}$ before and after filling. The inset images in (a), (b) and (c) show the normalized electric field densities on the holes before and after filling (e) Experimentally measured transmission spectrum from a red pixel fabricated with and without filling and the corresponding SEM images on left and right (f) SEM images of submicron RGB colour filters of different sizes after the filling scheme and the corresponding optical images show boundary errors eliminated at the pixel boundaries without any colour cross talk. The sub RGB filter sizes for the red are $1.95 \mu \mathrm{m} \times 5 \mu \mathrm{m}\left(\mathrm{RGB}_{1}\right), 875 \mathrm{~nm} \times 5 \mu \mathrm{m}\left(\mathrm{RGB}_{2}\right), 1.95 \mu \mathrm{m} \times 1.72 \mu \mathrm{m}$ $\left(\mathrm{RGB}_{3}\right)$ and $875 \mathrm{~nm} \times 975 \mathrm{~nm}\left(\mathrm{RGB}_{4}\right)(\mathrm{g}) \mathrm{SEM}$ image of an array of the submicron scale filters with the red pixel size of $875 \mathrm{~nm} \times 975 \mathrm{~nm}$ and the corresponding optical image shows three different colours red, green and blue (RGBs) without the filling scheme. The corresponding optical image shows the crescent shape of the red filter (the optical image follows its own SEM image geometry) and the boundary errors at the boundaries of each filter/pixel (h) SEM image of the same submicron scale filters after the filling scheme and the corresponding optical image showing the errors are eliminated $(\times 100$ magnification).

with a zig-zag shape at the filter boundaries of the submicron RGB filter. The pixel boundary errors at the edge of each submicron scale colour filter lead to macroscale pixel stitching errors when the RGB filters are juxtaposed with nanometre spacings.

These pixel boundary errors and the subsequent stitching errors cause blurring, irregularities and lack of sharpness when images are exhibited on displays or are projected onto screens. For submicron pixel sizes, this effect is significant and is a major challenge for practical display development as shown in Fig. 2c. Notably, these errors are present irrespective of the spacing between each submicron scale colour filters and are also present even if the pixels are joined seamlessly.
Elimination of the pixel boundary errors via a filling scheme. To circumvent the stitching issues for sub-micron pixels, we introduce a novel filling scheme. The filling scheme eliminates the errors by introducing extra holes into the colour filters as shown in Figs. 3a, $3 \mathrm{~b}$ and $3 \mathrm{c}$. The vacant spaces in the filter which cause the zig-zag irregularities at the boundaries are filled with holes with a suitable hole diameter and pitch; these produce a minimal effect on the resonance wavelength, and no colour deterioration. Finite element calculations were used to optimize the hole diameter and pitch for each pixel structure. The first step in the optimization is choosing a pitch for the inserted holes. The pitch is selected in such a way that the filter attains a more regular shape at the filter boundaries and 
such that the selected pitch does not introduce any new peaks or shifts in the resonance. Then the hole diameter used for the filling is varied to obtain the minimum boundary error. As the diameter of the added holes increases, minor peaks appear and also small shifts in the peak resonance occur. For the red filters, the boundary error is eliminated using a hole diameter of $170 \mathrm{~nm}$, placed at a distance $60 \mathrm{~nm}$ away (edge to edge) from the last hole in the horizontal direction and then vertically repeated (Fig. $3 \mathrm{a}$, left inset). In this way, the inserted holes and existing holes have a $372 \mathrm{~nm}$ pitch in the vertical direction and a $260 \mathrm{~nm}$ pitch in the horizontal direction. For the red, green and blue colour filters, $3 \times 4$ holes (filter size $1346 \mathrm{~nm} \times 1324 \mathrm{~nm}$ for red, $1005 \mathrm{~nm} \times 1037 \mathrm{~nm}$ for green and $770 \mathrm{~nm} \times 795 \mathrm{~nm}$ for blue) are used.

We have simulated the transmission spectrum for the red filter using the $3 \times 4$ hole structures (Fig. 3a) to make sure that there is no additional resonance (no colour cross-talk) created by the insertion of the filling holes. The same method has been followed for both the green and blue filters. For the green filter, the hole diameter used for filling is $90 \mathrm{~nm}$ with $285 \mathrm{~nm}$ and $195 \mathrm{~nm}$ pitch in the vertical and horizontal directions respectively (Fig. $3 \mathrm{~b}$ right inset). The blue filter is filled with $60 \mathrm{~nm}$ holes with pitch $225 \mathrm{~nm}$ vertically and $160 \mathrm{~nm}$ horizontally (Fig. $3 \mathrm{c}$ right inset). In the case of the red filter, the $260 \mathrm{~nm}$ pitch causes a small rise in transmission in the blue-green region as shown in Fig. 3a. For the green filter, the $195 \mathrm{~nm}$ pitch again produces a small peak towards the blue region of the spectrum. However this peak is outside the filter operating wavelength range $400 \mathrm{~nm}$ to $700 \mathrm{~nm}$ (Fig. 3b). For the blue filter, there is no extra peak created in the visible spectrum by the filling scheme (Fig. $3 \mathrm{c}$ ). The transmission reduces by $26 \%$ (red), $7 \%$ (green) and $6 \%$ (blue) of the peak (Fig. $3 \mathrm{a}-\mathrm{c}$ ) due to the presence of the defect, although the total number of holes is increased (Fig. 3a-d). The filling effect under the optical microscope $(50 \times$ magnification $)$ is more noticeable for the red filter than for the green or blue filters. This is because, for the red filters, the hole diameter and pitch used are bigger than those employed for the green and blue filters. However, small boundary errors are still present for the green and the blue pixels. For the red filter, the simulated transmission spectrum has been computed from the wavelength range $420 \mathrm{~nm}$ to $900 \mathrm{~nm}$ before and after filling (Fig. 3d) which confirms that there is no extra peak introduced by the filling scheme except for a weak feature in the blue region. Based on these simulation results, we have also fabricated a red filter with and without filling. Figure 3 shows the SEM image of the red filter before filling (left side of Fig. 3d) and after filling (right side of Fig. 3e). The inset shows the magnified SEM image to highlight that there is no overlapping of holes. Figure $3 \mathrm{e}$ shows the transmission spectrum of the fabricated red filter. This result matches the simulated spectrum for the red filter (Fig. 3d) except for a small peak very close to $400 \mathrm{~nm}$. This peak does not appear in the experimental plot because our spectrometer and light source used are not sensitive enough below $400 \mathrm{~nm}$ (U.V region). Since the pixel operates in the visible region, this peak will not cause any degradation in the colour. This peak can be pushed away from the visible region (400 $\mathrm{nm}$ to $700 \mathrm{~nm}$ ) by suitable optimization of the hole diameter and the pitch.

Based on the above simulations and experimental results, we have fabricated submicron RGB colour filters with no pixel boundary errors. Figure $3 f$ shows the SEM images and the corresponding optical images of the RGB colour filters. The images in Fig. $3 \mathrm{f}$ clearly show that there are no pixel boundary errors in comparison to Fig. $2 \mathrm{c}$ and also prove that the removal of such undesirable boundary effects is achieved with no colour cross talk. Furthermore we have fabricated a small array of the submicron filters to show the impact of the filling scheme. Fig. $3 g$ shows an array of sub RGB filters without the filling scheme, where the optical image of the red filter size is $875 \mathrm{~nm} \times$ $975 \mathrm{~nm}$. The optical image of the red submicron scale filter shows a crescent shape (the same as its own SEM image in Fig. 3g), and the boundary errors are visible for the green and blue submicron scale filters. The filling scheme can eliminate the errors as shown in Fig. 3h, where the crescent shape is converted to a rectangular shape. However, the colours observed at the submicron scale do not appear as bright as colours at the micron scale.

The filling scheme on a $430 \mathrm{~nm} \times 392 \mathrm{~nm}$ size colour filter. Based on results obtained from the above filling scheme, we have shown that the same filling scheme can be applied to a filter size of $430 \mathrm{~nm}$ $\times 392 \mathrm{~nm}$. The filter is designed using three holes with a hole diameter $150 \mathrm{~nm}$ and pitch $280 \mathrm{~nm}$ in a triangular fashion to obtain a resonance at $490 \mathrm{~nm}$. Fig. $4 \mathrm{a}$ and $4 \mathrm{~b}$ show the simulated $430 \mathrm{~nm} \times 392 \mathrm{~nm}$ colour filter with and without the filling. For the filling, the hole diameter used is $70 \mathrm{~nm}$ and pitch $195 \mathrm{~nm}$ as shown in the Fig. $4 \mathrm{~b}$. Fig. $4 \mathrm{c}$ shows the simulated transmission spectra from the $430 \mathrm{~nm} \times 392 \mathrm{~nm}$ colour filter with and without filling. The spectra show that even at the submicron scale, the optimized filling scheme does not introduce any prominent peaks or cause any colour cross talk or prominent shift in the peak wavelength. We have shown in Figs S3 and S4 of the Supplementary Information that study of the polarization insensitivity after the introduction of the filling scheme at submicron scale. Based on the simulation results, we have fabricated a submicron scale filter with size $430 \mathrm{~nm} \times 392 \mathrm{~nm}$ employing the filling scheme. Fig. $4 \mathrm{~d}$ and $4 \mathrm{e}$ show the SEM image of the fabricated submicron scale colour filter with size $430 \mathrm{~nm} \times$ $392 \mathrm{~nm}$ and the experimentally measured transmission spectrum, which shows the peak wavelength at $490 \mathrm{~nm}$. From Fig. 4e, it is clear that the experimentally obtained spectrum is in agreement with the simulated spectrum, both give the peak wavelength at $490 \mathrm{~nm}$ without introducing any new major peak or colour cross talk even after taking into account the fabrication tolerances (the diameter of holes used for the filling is a few nanometres wider than $70 \mathrm{~nm}$ ). The same scheme can be applied to both the red and green submicron filters. The diameter should be properly optimized for the filling scheme at the submicron scale as a very small diameter hole will not completely eliminate the boundary error while very big diameter holes can introduce a small shift or minor extra peak in the resonance. The results show that it is possible to overcome boundary errors at the submicron scale without colour cross talk using the filling scheme.

\section{Discussion}

We have used a simple, hole-based geometry to illustrate the importance of pixel boundary effects and stitching errors and we have also presented a filling scheme for correcting this effect. However, the hole-based, submicron-scale filter design is angle dependent i.e., the transmission wavelength depends on the angle of incidence of light impinging on the filter. If the hole array were used in a display, the filter would transmit the designed colour at normal incidence while different colours would be filtered at oblique incidence. Figure S5a in the Supplementary Information shows the simulated shift in the resonance peak with respect to the angle of incidence for a hole based green filter. Also, for the hole based design, different colours are obtained by varying mainly the pitch and to a lesser extent the hole diameter. This variation in the pitch necessitates different sizes for each RGB filter when a submicron filter is designed using a three hole geometry as shown in the Figs. $3 \mathrm{f}$ and $3 \mathrm{~g}$. Due to the fact that the surface plasmon resonance depends on the pitch, the extra holes used for filling do cause a small reduction in the transmission of the filter, as shown in Fig. 4c.

To overcome all the above issues, and satisfy all the requirements for submicron scale filter development, we have developed coaxial hole $(\mathrm{CH})$ based submicron filters in aluminium by combining and tuning surface plasmon resonance with Fabry-Perot resonances, which meet all the desired properties including angle insensitivity. 

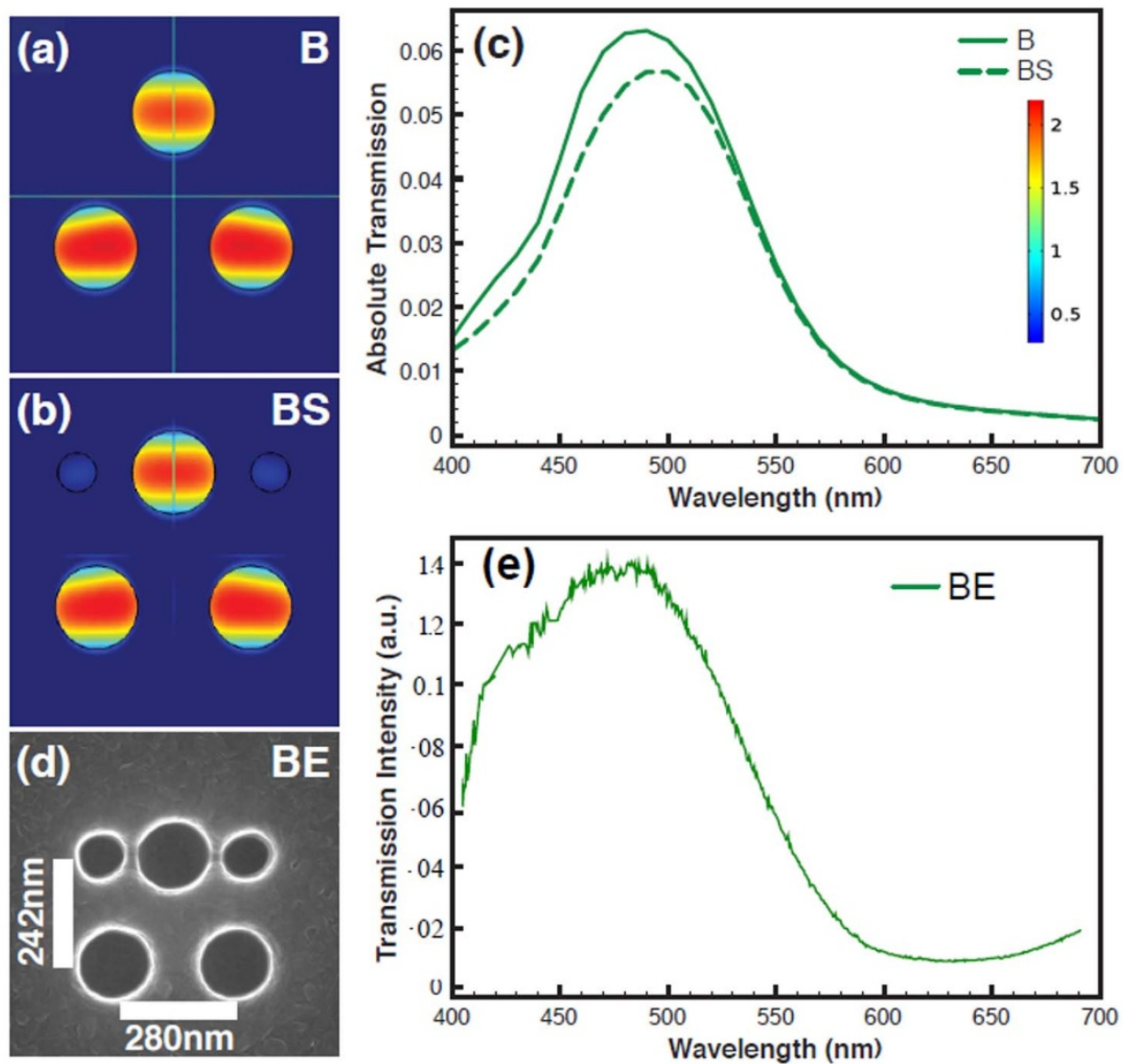

Figure 4 A submicron scale pixel with size $430 \mathrm{~nm} \times 392 \mathrm{~nm}$ with and without filling scheme (a) The simulated submicron scale colour filter with size $430 \mathrm{~nm} \times 392 \mathrm{~nm}$ in Al using an equilateral triangular geometry (hole diameter $150 \mathrm{~nm}$, pitch $280 \mathrm{~nm}$ and Al film thickness $130 \mathrm{~nm}$ ). The figure shows normalized electric field (E-field) densities on holes (b) The same filter after filling scheme using $70 \mathrm{~nm}$ diameter holes (c) Simulated transmission spectra from the same submicron scale colour filter with and without filling (d) SEM image of the fabricated submicron scale colour filter based on the simulation results with size $430 \mathrm{~nm} \times 392 \mathrm{~nm}$ in Al using a triangular fashion after filling (e) Experimentally obtained transmission spectra from the fabricated $430 \mathrm{~nm} \times 392 \mathrm{~nm}$ submicron scale filter.

In a $\mathrm{CH}$ plasmonic filter, the transmission peaks are caused by Fabry-Perot resonances and planar surface plasmons (PSPs) ${ }^{23,24}$. However, the resonance peak wavelength is determined largely by the Fabry-Perot resonance condition ${ }^{23-28}$. The Fabry-Perot resonance is dependent on the geometry of the holes and thickness of the film, while the surface plasmon resonance is dominated by the pitch of the two dimensional gratings. In the case of a $\mathrm{CH}$ filter, the transmission peaks are dominated by Fabry-Perot resonances in the cylindrical cavity (cylindrical surface plasmons) of the metal film with finite thickness and two end faces. The desired transmission peak can be estimated using the equation $l=(\mathrm{n} \pi-\theta) / \beta^{27}$, where $l$ is the thickness of the metal film, $n$ is the order of the Fabry-Pérot resonance, $\theta$ is the phase of reflection constant and $\beta$ is the propagation constant. By keeping the metal thickness $(l)$ constant, the propagation constant $(\beta)$ can be varied with different $\mathrm{CH}$ aperture sizes to tune the transmission peak. Since the Fabry-Perot resonances are associated with localized surface plasmons ${ }^{29}$, there is no shift in the resonance peak with respect to the angle of incidence for the $\mathrm{CH}$ based filters (Fig. S5b in the Supplementary information). In the $\mathrm{CH}$ based filter design, tuning of the resonance peaks (colours) is achieved by varying the inner $\left(R_{1}\right)$ and outer radius $\left(R_{2}\right)$ of the coaxial hole while keeping the pitch the same. In addition to all of these advantages of $\mathrm{CH}$ based filters, the boundary error can be completely eliminated with no colour cross talk and transmission intensity reduction. This is because the filling doesn't affect the res- onance much due to its pitch because the resonance is dominated by the Fabry-Perot effect, which is independent of pitch.

Submicron scale colour filters using $\mathrm{CH}-\mathrm{CH}$ filling combination. A red colour filter is obtained by choosing $R_{1}=130 \mathrm{~nm}$ and $R_{2}=$ $100 \mathrm{~nm}\left(\mathrm{CHR}_{1}\right)$ to obtain a peak wavelength at $690 \mathrm{~nm}$ (Fig. 5a). The pitch is chosen to be $430 \mathrm{~nm}$ because this pitch also corresponds to resonance towards the red region of the visible spectrum. So a red submicron filter is designed $\left(\mathrm{CHR}_{1}\right)$, which meets all the criteria including the angle insensitivity, except the filter boundary error as shown in Fig. 5a. Here, to eliminate the filter boundary error, another $\mathrm{CH}$ geometry is designed with $\mathrm{R}_{1}=90 \mathrm{~nm}$ and $\mathrm{R}_{2}=70 \mathrm{~nm}\left(\mathrm{CHR}_{2}\right)$, which is smaller than the $\mathrm{CHR}_{1}$ as shown in Fig. 5b. Both the $\mathrm{CHR}_{1}$ and $\mathrm{CHR}_{2}$ designs yield peak wavelengths of $690 \mathrm{~nm}$. Then the $\mathrm{CHR}_{2}$ is used for filling the $\mathrm{CHR}_{1}$ filter to create a new combination filter, which we denote ' $\mathrm{CHR}_{2}+\mathrm{CHR}_{1}$ ', as shown in Fig. $5 \mathrm{c}$. Figure $5 \mathrm{~g}$ shows the computationally obtained peak with only $\mathrm{CHR}_{1}, \mathrm{CHR}_{2}$ and with the $\mathrm{CHR}_{2}+\mathrm{CHR}_{1}$ combination.

The filter boundary error is fully eliminated with no major additional peaks and also with enhancement in the transmission intensity. The $\mathrm{CH}-\mathrm{CH}$ based filling scheme is ideal because $\mathrm{CHR}_{2}$ used for filling doesn't affect the resonance much due to its pitch because the resonance is dominated by the Fabry-Perot effect, which is independent of pitch. To demonstrate this experimentally, we have fabricated a red colour filter based on the $\mathrm{CH}$ combination. Figure $5 \mathrm{~d}$ shows the 


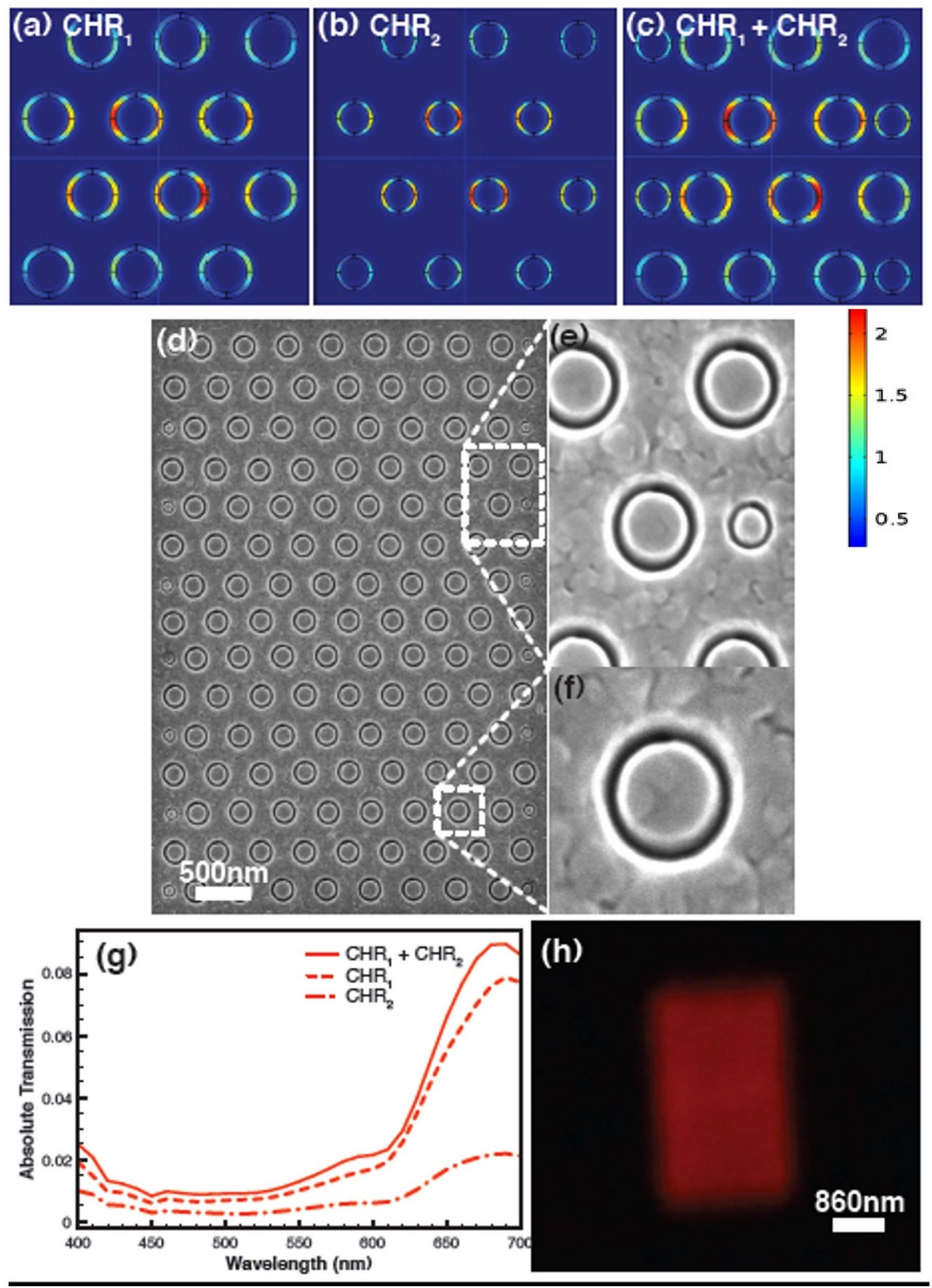

Figure $5 \mid$ Filling scheme for the coaxial hole $(\mathrm{CH})$ based filter (a) Simulated red colour filter $\mathrm{CHR}_{1}$ shows normalized electric field (E-field) with the boundary error (b) Simulated submicron red colour filter $\mathrm{CHR}_{2}$ shows normalized electric field with the boundary error (c) Combination of CHR and $\mathrm{CHR}_{1}$, ie $\mathrm{CHR}_{2}+\mathrm{CHR}_{1}$ showing normalized electric field, where the boundary error is fully eliminated (d) SEM image of the fabricated red colour filter $\mathrm{CHR}_{2}+\mathrm{CHR}_{1}$ (e) Magnified SEM image of the $\mathrm{CHR}_{2}+\mathrm{CHR}_{1}$ showing $\mathrm{CHR}_{1}$ and $\mathrm{CHR}_{2}$ (f) Magnified SEM image of one CH (g) Computationally obtained transmission spectra from $\mathrm{CHR}_{1}, \mathrm{CHR}_{2}$ and $\mathrm{CHR}_{2}+\mathrm{CHR}_{1}$ combination (h) Optical image of the filter $\mathrm{CHR}_{2}+\mathrm{CHR}_{1}$ combination shows no pixel boundary error. 

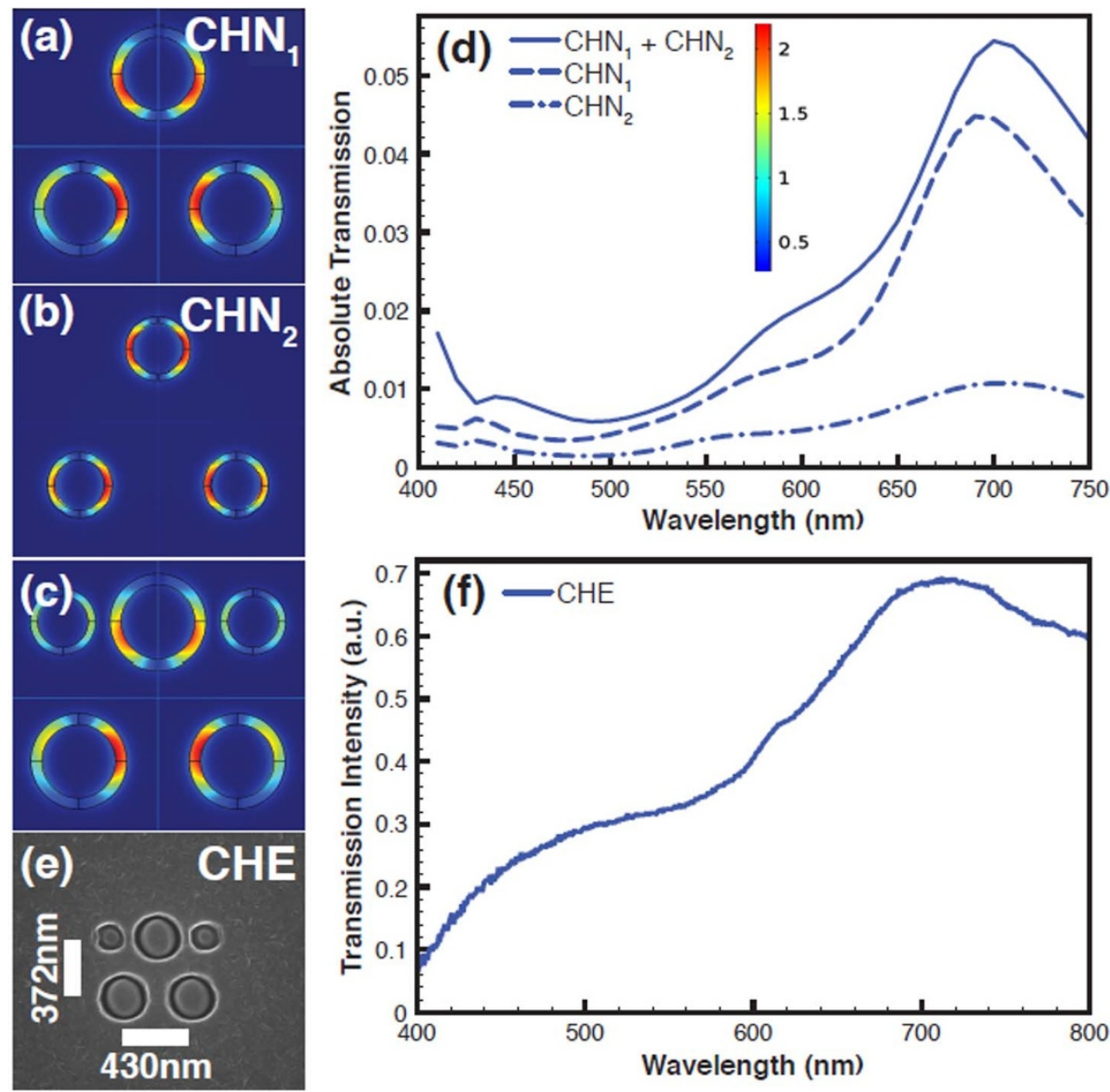

Figure 6 | A CH-CH combination based submicron scale filter with size $690 \mathrm{~nm} \times 632 \mathrm{~nm}$ (a) The simulated CH submicron scale colour filter with size $690 \mathrm{~nm} \times 632 \mathrm{~nm}$ in an equilateral triangular geometry using three CHs with the inner and outer radii of $130 \mathrm{~nm}$ and $100 \mathrm{~nm}$ respectively and the pitch of $430 \mathrm{~nm}\left(\mathrm{CHN}_{1}\right)$ (the figure shows normalized electric field (E-field) on the $\mathrm{CHs}$ ) (b) $\mathrm{CHN}_{2}$ with the inner and outer radii of $90 \mathrm{~nm}$ and $70 \mathrm{~nm}$ (c) The filter after the $\mathrm{CH}-\mathrm{CH}$ combination scheme (d) Simulated transmission spectra from the $\mathrm{CH}$ submicron scale colour filters $\left(\mathrm{CHN}_{1}\right.$, $\mathrm{CHN}_{2}$ and $\mathrm{CHN}_{1}+\mathrm{CHN}_{2}$ combination (e) SEM image of the fabricated CH based submicron scale colour filter CHE with size $690 \mathrm{~nm} \times 632 \mathrm{~nm}$ in $\mathrm{Al}$ using $\mathrm{CHN}_{1}-\mathrm{CHN}_{2}$ combination (f) Experimentally obtained transmission spectrum from the CHE with size $690 \mathrm{~nm} \times 632 \mathrm{~nm}$.

SEM image of the $\mathrm{CHR}_{2}+\mathrm{CHR}_{1}$ combination filter. Figures $5 \mathrm{e}$ and $5 \mathrm{f}$ show the magnified SEM image of the filter. Figure 5 h shows an optical image of the $\mathrm{CHR}_{1}+\mathrm{CHR}_{2}$ combination filter showing no filter stitching error and colour cross talk at the boundaries. The same method can be repeated for the green and blue colour filters.

The CH-CH filling combination on $690 \mathrm{~nm} \times 632 \mathrm{~nm}$ size filter. As a next step, we show the $\mathrm{CH}-\mathrm{CH}$ based filling scheme applied to a submicron filter using the equilateral triangular geometry with a size $690 \mathrm{~nm} \times 632 \mathrm{~nm}$ (Fig. 6). As shown in Fig. 6a, the inner and outer radii are tuned to $130 \mathrm{~nm}$ and $100 \mathrm{~nm}$ respectively and the pitch to $430 \mathrm{~nm}$ in order to obtain a resonance in the red region at $690 \mathrm{~nm}$ $\left(\mathrm{CHN}_{1}\right)$. For filling, as mentioned in the above section a small $\mathrm{CH}$ with outer and inner radii $90 \mathrm{~nm}$ and $70 \mathrm{~nm}$ is used as shown in Fig. $6 \mathrm{~b}\left(\mathrm{CHN}_{2}\right)$. Then $\mathrm{CHN}_{1}$ and $\mathrm{CHN}_{2}$ are combined to create the submicron scale filter $\mathrm{CHN}_{1}+\mathrm{CHN}_{2}$ as shown in the Fig. 6c. Fig. $6 \mathrm{~d}$ shows the computationally obtained spectra from the submicron scale filter with and without the $\mathrm{CH}-\mathrm{CH}$ filling scheme. The spectra show that the $\mathrm{CH}-\mathrm{CH}$ filling scheme does not introduce any new peaks, nor does it cause any colour cross talk, even for a filter size of $690 \mathrm{~nm} \times 632 \mathrm{~nm}$.

The transmission intensity increases after the filling scheme is implemented as shown in Fig. 6d. We have shown polarization insensitivity study of the $\mathrm{CH}$ triangular geometry at the submicron scale in S6 of the Supplementary information. Based on the simu- lation results, we have fabricated a submicron scale filter with the size $690 \mathrm{~nm} \times 632 \mathrm{~nm}$ using the $\mathrm{CH}-\mathrm{CH}$ filling scheme. The SEM image of the filter is shown in Fig. 6e (CHE). Fig. $6 \mathrm{f}$ shows the experimentally measured transmission spectrum from the $690 \mathrm{~nm} \times 632 \mathrm{~nm}$ size filter, which exhibits a peak at $690 \mathrm{~nm}$ and this matches the simulation results, except for the presence of two minor peaks at $400 \mathrm{~nm}$ and $570 \mathrm{~nm}$. These peaks can be suppressed further by tuning the pitch and the $\mathrm{CH}$ geometry. Using the $\mathrm{CH}-\mathrm{CH}$ filling scheme, each submicron scale RBG filter can be made of the same size because the colour (the resonance) is tuned mainly by varying the gap between outer and inner radii keeping the pitch same. These results show that the $\mathrm{CH}-\mathrm{CH}$ filling scheme is the best strategy to develop submicron colour filters with uniform size for each RGB filter without stitching errors, which are also polarization independent, angle insensitive and based on LCD compatible aluminium technology. For large area fabrication, electron beam lithography or nanoimprint lithography can be used, so that the filling scheme can be easily integrated into the design.

In summary, we have presented a pathway for the development of submicron plasmonic colour filters. We initially determined the minimum plasmonic filter size required to define a submicron scale pixel using simple holes in aluminium. The results showed that the minimum number of holes required for realizing a polarization independent submicron colour filter is three in an equilateral triangular arrangement. This enables development of triangular pixels in dis- 
plays. But the majority of state-of-the-art displays use rectangular or square shape pixels. We then demonstrated that development of rectangular or square shape pixels is hindered by boundary errors and stitching errors at the submicron scale. These errors cause blur, reduced sharpness and discontinuities in the displayed images. In order to eliminate these errors, we have developed a simple but powerful filling strategy by combining surface plasmon and FabryPerot resonances. Using both computational methods and experiments, a submicron scale colour filter of $430 \mathrm{~nm} \times 392 \mathrm{~nm}$ size was fabricated without any colour cross talk or pixel boundary errors. We then discussed drawbacks of hole based designs as they are angle sensitive with transmission wavelength depending on the angle of incidence. Furthermore, it is difficult to obtain the same filter size for each RGB filter at the submicron scale because the pitch is varied in order to generate different colours, and the filling scheme reduces the transmission intensity. In order to address all these issues, we developed the $\mathrm{CH}-\mathrm{CH}$ filling combination scheme and demonstrated a submicron scale filter with the size $690 \mathrm{~nm} \times 632 \mathrm{~nm}$, which completely eliminated the boundary and stitching errors with increased transmission without any colour cross talk. In addition, these filters are polarization independent, angle insensitive and based on LCD compatible aluminium technology. These submicron colour filters may have applications in displays, projectors, spatial light modulators (SLM), liquid crystal over silicon technology (LCOS) and wavefront sensors.

\section{Methods}

Focused ion beam (FIB). A glass wafer of thickness $500 \mu \mathrm{m}$ was used for fabricating the submicron scale plasmonic filters for different filter geometries. The glass substrate was cleaned using acetone, isopropyl alcohol and deionized water. A $150 \mathrm{~nm} \mathrm{Al} \mathrm{film} \mathrm{was} \mathrm{evaporated} \mathrm{at} \mathrm{the} \mathrm{rate} \mathrm{of} 0.5 \AA / s$ on the wafer using Intlvac Nanochrome II electron beam evaporation system. Both the coaxial holes (CHs) and the holes were milled into the Al using an FEI Helios NanoLab 600 Dual Beam focused ion beam (FIB). For holes, the current was varied from $1.5 \mathrm{pA}$ to $28 \mathrm{pA}$ depending on the feature size by setting the voltage at $30 \mathrm{kV}$. The sample was loaded immediately from the evaporator to the FIB to avoid the Al being oxidized. After the milling the sample was immediately coated with $50 \mathrm{~nm} \mathrm{SiO}_{2}$ using plasma enhanced chemical vapor deposition (PECVD) to prevent the $\mathrm{Al}$ from oxidizing and for matching the index with the glass substrate to enhance transmission. We have fabricated the same feature at different location of the sample and also in different samples. We have observed the same colour from all these features. From this, we understand that the a few submicron roughnesse will not shift the resonance to change any colour. For the coaxial hole $(\mathrm{CH})$ array, the $\mathrm{Al}$ was evaporated at the rate of $0.4 \AA$ /s followed by milling using the FIB. The current used for the milling was $1.5 \mathrm{pA}$ and the voltage $30 \mathrm{kV}$. The resonance peak of the $\mathrm{CH}$ array was very sensitive to any dielectric film on the top. The dielectric sensitivity of the resonance peak for the $\mathrm{CH}$ filters can be used for further tuning the peak wavelength position by depositing materials such as $\mathrm{MgF}_{2}$ (refractive index $\sim 1.38$ ) or $\mathrm{SiO}_{2}$ (refractive index $\sim 1.5$ ). We have taken cross section of the fabricated arrays after the milling to make sure that $150 \mathrm{~nm} \mathrm{Al}$ was milled through. We have done this after depositing platinum on the structure to protect the structure while taking a cross section and getting better contrast between the glass and Al. We have also used $130 \mathrm{~nm}$ thick Al film for making submicron filters.

Optical characterization. The white excitation light from a halogen lamp (100 W) was focused onto the sample using a Nikon TE2000-S Eclipse inverted microscope. The transmitted light through the sample was collected via a $40 \times$ dry objective lens with an iris diaphragm (numerical aperture 0.6) and then focused onto the entrance slit of a MicroSpec $2150 \mathrm{i}$ imaging spectrometer coupled to a thermoelectrically cooled $\left(-60^{\circ} \mathrm{C}\right) \mathrm{CCD}$ (charge-coupled device) (Acton Pixis 1024B Exelon). To get spectra from small feature sizes ( $<1$ micron) a fiber based spectrometer to measure the transmission percentage from submicron features. We have used collimated illumination and different NAs $(\sim 0.3-0.7)$ depending on the feature size. The spectrum for the coaxial geometry was same for all NAs, which is due to the angle insensitiveness of the geometry. The low image brightness of the features at submicron scale is due to the fact that they are beyond the resolution of an optical microscope. For the regular holes, transmission in the order $7-35 \%$ was observed (35\% for large red colour filters $\sim 8 \mu \mathrm{m}$ and close to $7-10 \%$ for submicron red colour filters). For the coaxial holes, transmission varied between $5-62 \%$, again the transmission reduced to $5-8 \%$ for submicron filters.

Numerical simulations. The submicron scale plasmonic filters were computationally investigated using the finite element method (FEM) implemented in COMSOL MULTIPHYSICS. For hole based colour filters, the pitch and hole diameter was varied to get different colours. The simulation model to find the peak wavelength of the colour filters consists of $150 \mathrm{~nm}$ or $130 \mathrm{~nm} \mathrm{Al}$ on a semi-infinite thick glass substrate. The $\mathrm{Al}$ was then covered with $50 \mathrm{~nm}$ silica $\left(\mathrm{SiO}_{2}\right)$ and then semi-infinite air above the silica. We have used a finite number of plasmonic elements (simple holes or coaxial holes) in the $\mathrm{Al}$ and perfectly matched layer (PML) was used on four side walls. The light excitation was done from the substrate side. We have used Sparameters to find the transmission percentage $\left(\mathrm{S}_{21}\right)$. The same model was used for $\mathrm{CH}$ based colour filters. For the $\mathrm{CH}$ based filters, the simulations were carried out with $\mathrm{SiO}_{2}$ and without $\mathrm{SiO}_{2}$ layer on the top of the Al. There was a considerable red shift in the peak with $\mathrm{SiO}_{2}$ for the $\mathrm{CH}$ based filters. The filters can also be fabricated without a $\mathrm{SiO}_{2}$ layer as a cap layer on the top of the $\mathrm{Al}$. Then the optimization of parameters should be done using the effective refractive as mentioned in the Ref. [13] to take into account the oxide layer $\left(\mathrm{Al}_{2} \mathrm{O}_{3}\right)$. A submicron colour filter with sizes $430 \mathrm{~nm} \times$ $392 \mathrm{~nm}$ (for holes based design) and $690 \mathrm{~nm} \times 632 \mathrm{~nm}$ (for the $\mathrm{CH}-\mathrm{CH}$ based design) were designed using 3 holes and $\mathrm{CHs}$ in a triangular geometry.

1. Sabnis, R. W. Color filter technology for liquid crystal displays. Displays 20, 119-129 (1999).

2. Pyayt, A. L., Starkweather, G. K. \& Sinclair, J. M. A high-efficiency display based on a telescopic pixel design. Nature Photonics 2, 492-495 (2008).

3. Tsuda, K. Colour filters for LCDs. Displays 14, 115-124 (1993).

4. Hsu, T. H., Fang, Y. K., Lin, C. Y., Chen, S. F., Lin, C. S., Yaung, D. N., Wuu, S. G., Chien, H. C., Tseng, C. H., Lin, J. S. \& Wang, C. S. Light guide for pixel crosstalk improvement in deep submicron CMOS image sensor. IEEE Electron Device Lett. 25, 22-24 (2004).

5. Yokogawa, S., Burgos, S. P. \& Atwater, H. A. Plasmonic Color Filters for CMOS Image Sensor Applications. Nano Letters 12, 4349-4354 (2012).

6. Wu, Y.-K., Hollowell, A. E., Zhang, C. \& Guo, L. J. Angle-Insensitive Structural Colours based on Metallic Nanocavities and Coloured Pixels beyond the Diffraction Limit. Sci. Rep. 3, 1194 (2013).

7. Ebbesen, T. W., Lezec, H. J., Ghaemi, H. F., Thio, T. \& Wolff, P. A. Extraordinary optical transmission through subwavelength hole arrays. Nature 391, 667-669 (1998).

8. Kumar, K., Duan, H., Hegde, R. S., Koh, S. C. W., Wei, J. N. \& Yang, J. K. W Printing colour at the optical diffraction limit. Nature Nanotech. 7, 557-561 (2012).

9. Zeng, B., Gao, Y. \& Bartoli, F. J. Ultrathin Nanostructured Metals for Highly Transmissive Plasmonic Subtractive Color Filters. Sci. Rep. 3, 2840 (2013).

10. Barrow, S. J., Wei, X., Baldauf, J. S., Funston, A. M. \& Mulvaney, P. The Surface Plasmon Modes of Self-Assembled Gold Nanocrystals. Nat. Commun. 3, 1275 (2013).

11. Kotnala, A. \& Gordon, R. Quantification of High-Efficiency Trapping of Nanoparticles in a Double Nanohole Optical Tweezer. Nano Letters 14 (2), 853-856 (2014).

12. West, P. R., Ishii, S., Naik, G. V., Emani, N. K., Shalaev, V. M. \& Boltasseva, A. Searching for better plasmonic materials. Laser Photonics Rev 4, 795-808 (2010).

13. Knight, M. W., King, N. S., Liu, L., Everitt, H. O., Nordlander, P. \& Halas, N. J. Aluminum for Plasmonics. ACS Nano 8 (1), 834-840 (2014).

14. Catrysse, P. B. \& Wandell, B. A. Integrated colour pixels in 0.18 um complementary metal oxide semiconductor technology. Journal of the Optical Society of America A 20, 2293-2306 (2003).

15. Inoue, D., Miura, A., Nomura, T., Fujikawa, H., Sato, K., Ikeda, N., Tsuya, D., Sugimoto, Y. \& Koide, Y. Polarization independent visible color filter comprising an aluminum film with surface-plasmon enhanced transmission through a subwavelength array of holes. Appl. Phys. Lett. 98, 093113 (2011).

16. Liu, W., Sukhorukov, A. A., Miroshnichenko, A. E., Poulton, C. G., Xu, Z., Neshev, D. N. \& Kivshar, Y. S. Complete spectral gap in coupled dielectric waveguides embedded into metal. Appl. Phys. Lett. 97, 021106 (2010).

17. Chen, Q. \& Cumming, D. R. S. High transmission and low color cross-talk plasmonic color filters using hexagonal-lattice hole arrays in aluminum films. Opt. Express 18, 14056 (2010).

18. Najiminaini, M., Vasefi, F., Kaminska, B. \& Carson, J. J. L. Nanohole-array-based device for 2D snapshot multispectral imaging. Sci. Rep. 3, 2589 (2013).

19. Lee, H. S., Yoon, Y. T., Lee, S. S., Kim, S. H. \& Lee, K. D. Color filter based on a subwavelength patterned metal grating. Opt. Express 15, 15457-15463 (2007).

20. Lezec, H. J. \& Thio, T. Diffracted evanescent wave model for enhanced and suppressed optical transmission through subwavelength hole arrays. Opt. Express 12, 3629-3651 (2004).

21. Burgos, S. P., Yokogawa, S. \& Atwater, H. A. Color Imaging via Nearest Neighbor Hole Coupling in Plasmonic Color Filters Integrated onto a Complementary Metal-Oxide Semiconductor Image Sensor. ACS Nano 7 (11), 10038-10047 (2013).

22. Chen, Q., Chitnis, D., Walls, K., Drysdale, T. D., Collins, S. \& Cumming, D. R. S. CMOS photodetectors integrated with plasmonic color filters. IEEE Photonics Technol. Lett. 24, 3 (2012).

23. Haftel, M. I., Schlockermann, C. \& Blumberg, G. Enhanced transmission with coaxial nanoapertures: Role of cylindrical surface plasmons. Phys. Rev. B 74, 235405 (2006).

24. Roberts, A. Beam transmission through hole arrays. Opt. Express 18, 2528-2533 (2010).

25. Heshmat, B., Li, D., Darcie, T. E. \& Gordon, R. Tuning plasmonic resonances of an annular aperture in metal plate. Opt. Express 19, 5912-5923 (2011). 
26. Salvi, J., Roussey, M., Baida, F. I., Bernal, M.-P., Mussot, A., Sylvestre, H. T., Maillotte, D., Van Labeke, D., Perentes, A., Utke, I., Sandu, C., Hoffmann, P. \& Dwir, B. Annular aperture arrays: study in the visible region of the electromagnetic spectrum. Opt. Lett. 30, 1611-1613 (2005).

27. Si, G., Zhao, Y., Liu, H., Teo, S., Zhang, M., Huang, T. J., Danner, A. J. \& Teng, J. Annular aperture array based color filter. Appl. Phys. Lett. 99, 033105 (2011)

28. Poujet, Y., Salvi, J. \& Baida, F. I. 90\% Extraordinary optical transmission in the visible range through annular aperture metallic arrays. Opt. Lett. 32, 2942-2944 (2007).

29. Langhammer, C., Schwind, M., Kasemo, B. \& Zorić, I. Localized Surface plasmon resonancein Aluminum Nanodisks. Nano Lett. 8, 1461-1471 (2008).

\section{Acknowledgments}

This research was supported by a UM Interdisciplinary Seed Grant and the McKenzie Fellowship Scheme of The University of Melbourne. The authors also acknowledge funding received from the Melbourne Materials Institute (MMI) through the MMI-MCN merit time allocation scheme. AR acknowledges ARC support through Grant DP110100221. ST-H acknowledges her ARC ARF fellowship DP1096288. And PM thanks the ARC for support through LF100100117. The authors would like to acknowledge Fatima Eftekhari, Stuart Earl and Daniel Gomez for discussions. This work was performed in part at the Melbourne Centre for Nanofabrication (MCN) in the Victorian Node of the Australian National Fabrication Facility (ANFF). The numerical simulations were undertaken on the NCI National Facility in Canberra, Australia, which is supported by the Australian Commonwealth Government.

\section{Author contributions}

R.R. conceived and implemented the idea and directed the project. R.R. prepared the sample. K.G. and S.K. assisted the sample preparation. R.R. and E.B. fabricated the device using FIB. S.C., A.M., R.R., D.N. and A.D.A. designed the experiments. A.M., S.C., R.R. and A.D.A. carried out spectral measurements. R.R. carried out simulations. A.R., A.D.A., S.T.-H. and T.J. contributed to simulations. R.R. and T.J. prepared the graphics. R.R., K.G. and A.D.A. carried out microscope experiments. R.R. wrote the paper and A.R., P.M., D.N., E.S., S.T.-H. and S.P. participated in the analysis of results, discussing and writing the manuscript. A.R., E.S., P.M., D.N. and S.P. supervised the research. All authors reviewed the manuscript.

\section{Additional information}

Supplementary information accompanies this paper at http://www.nature.com/ scientificreports

Competing financial interests: The authors declare no competing financial interests. How to cite this article: Rajasekharan, R. et al. Filling schemes at submicron scale: Development of submicron sized plasmonic colour filters. Sci. Rep. 4, 6435; DOI:10.1038/ srep06435 (2014).

This work is licensed under a Creative Commons Attribution 4.0 International License. The images or other third party material in this article are included in the article's Creative Commons license, unless indicated otherwise in the credit line; if the material is not included under the Creative Commons license, users will need to obtain permission from the license holder in order to reproduce the material. To view a copy of this license, visit http://creativecommons.org/licenses/by/4.0/ 